\title{
Need for Gender Focused Policies Addressing Smokeless Tobacco Use among Women in India: A Review
}

\author{
A G Radhika ${ }^{1 *}$, G S Preetha ${ }^{2}$, Sutapa Bandyopadhyay Neogi²
}

\begin{abstract}
Background: Nearly 8 million annual deaths occurring globally are attributable to tobacco use. Among more than 356 million smokeless tobacco (SLT) users in 140 countries, $82 \%$ reside in Southeast Asia with the vast majority being in India and Bangladesh. According to the Global Adult Tobacco Survey -2 data, 21.4\% of adults in India consume SLT, among them $29.6 \%$ are men and $12.1 \%$ women. SLT has received less attention compared to its smoked counterparts in the public health measures to curb tobacco use. Though women are a sizable proportion of users, majority of the awareness building measures as well as governmental policies do not target them. This review aims to highlight these gaps objectively with constructive suggestions to enable a changed strategy to reduce tobacco consumption. Aim: (1) To critically review the gender sensitivity of tobacco control measures in India, (2) to conduct a comparative analysis of gender responsive strategies in India with those in smokeless tobacco high burden countries and (3) to make practical, feasible recommendations to enhance gender responsiveness of tobacco control measures in India in general and smokeless tobacco in particular. Methodology and Results: Following a comprehensive literature review to capture key information on gender responsiveness/sensitivity of strategies for tobacco control publications in English within the last 20 years, our search yielded 35 papers and reports from India describing policies relevant to SLT and women. Public health approach to tobacco control in general was found to be gender blind. Conclusion: It is evident that tobacco and smokeless tobacco related information and awareness activities need to focus more on women with improved messaging strategy to make it easily understandable and tailor the same to address the immediate and delayed health concerns. This much needed change would receive impetus with revisions in Governmental tobacco control policies, implementation and uptake.
\end{abstract}

Keywords: Non-smoked tobacco- gender- women- policy- tobacco control policy

Asian Pac J Cancer Prev, 22, Progress of Tobacco Control in the South-East Asia Region Suppl, 7-12

\section{Introduction}

Tobacco use in smoked and smokeless forms, accounts for $10 \%$ of all deaths, worldwide, numbering to 8 million annually, most of them in developing countries (Sinha et al., 2018). Globally, in 2016, about $34 \%$ of men and $6 \%$ of women aged more than 15 years smoked tobacco whereas $8 \%$ men and $5 \%$ women in the same age group used smokeless tobacco (SLT). Among school-going children aged $13-15$ years, $9 \%$ of boys and $4 \%$ of girls smoked cigarettes, while $5 \%$ of boys and $3 \%$ of girls used smokeless tobacco products (WHO, 2019). Among more than 356 million SLT users in 140 countries, 82\% reside in Southeast Asia with the vast majority in India and Bangladesh (Sinha et al., 2018).

The various forms of tobacco used in Southeast Asia region include cigarettes, bidis (dried tobacco rolled in paper or leaf), SLT such as khaini (tobacco with slaked lime and aromatic spices), surti (dried tobacco leaves for chewing), or paan masala (tobacco with aromatic spices), gutkha (mixture of tobacco and molasses available in small sachets), application of gul or gudaku as dentifrice, and inhaling Nas and Naswar (nasal inhalation of tobacco powder) (Sreeramareddy et al., 2014). Majority of these products contain extremely high levels of nicotine resulting in chronic dependence. (IARC WG, 2007)

India is the second largest producer of tobacco in the world after China. According to the Global Adult Tobacco Survey -2 ( GATS 2) data, $21.4 \%$ of adults in India consume SLT, among them $29.6 \%$ are men and $12.1 \%$ women. Majority (three-fourth) of these adults reside in rural areas (GATS-2., 2017). Women in rural areas or those who were less educated and from low socio-economic strata were found to use SLT more often (Sinha et al., 2012). SLTs like gul, gudaku, mishri, masher, lal dantamanjan (dentrifice), and nas/naswar are common in Bangladesh, India, and Nepal (Sreeramareddy et al., 2014). Seventy million women use SLT in India, with many reporting that they used SLT to suppress hunger during laborious tasks (Gupta et al., 2016). 
Although 181 countries and the European Union have ratified the WHO Framework Convention for Tobacco Control (FCTC), the global focus has remained mainly on curbing cigarette smoking. SLT prevention and control measures have been largely overlooked (Mehrotra et al., 2019). The deleterious effects of SLT have been well documented, however it has received much less attention in policies or programmes (Onoh et al., 2021).

Nicotine produced from tobacco metabolism affects almost all organs in the body resulting in adverse health outcomes like oropharyngeal, oesophageal, and pancreatic cancers; pre-malignant and malignant lesions in oral cavity; diabetes mellitus; cardiovascular diseases; osteopenia ; mental illness; low birth weight; preterm births; small for gestation age babies and stillbirths (Onoh et al., 2021). The consequences of SLT use affect women disproportionately with nearly three fifths of deaths attributable to SLT use in women (Onoh et al., 2021).

Global tobacco control efforts including the LMICs, tend to be gender-neutral. Dearth in the response of the tobacco control efforts in applying gender and diversitybased interventions requires immediate correction given with the comprehensive gendered approach of the tobacco industry. Direct as well as surrogate advertisement for tobacco products (particularly the smoked form) at point of sale, television, print, internet, and social media are rampant (Siddiqi et al., 2015). The tobacco companies have developed newer and more economic tobacco products for women e.g. lipstick tube shape, slim and super slim cigarettes available in Russia.

In India, SLT products are produced both commercially by tobacco companies and by informal small cottage industry. SLTs produced by former are sold in attractive packages with surrogate advertisements of non- tobacco containing products (Arora et al., 2010). Involvement of both formal and informal sector in SLT production and sales pose a challenge to the control measures. Leading cigarette companies are entering the smokeless tobacco market, perhaps because of continuing declines in cigarette consumption and increases in smoke-free ordinances.

Smokeless tobacco is also covered by the World Health Organization-Framework Convention on Tobacco Control (WHO-FCTC), the international treaty signed by 181 countries. The packaging and labelling of tobacco products are considered effective tobacco control measures and are adopted as a provision of WHO-FCTC in article 11 (Mehrotra et al., 2019). SLT-targeted campaigns in print and electronic media, educational programs, and other related interventions are lacking in most of the FCTC signatory countries including India. All forms of tobacco have detrimental effects on endocrinological and reproductive health too but anti-smoking campaigns and graphic warnings in India usually target general health concerns and include warnings for cancers (Raute et al., 2009) e.g. lung and oral malignancies, that occur late in life.

These points highlight the need to implement tobacco prevention and cessation programmes that target the tobacco products that Indian population, especially the use among women. It is also important to improve the messaging strategy to make it easily understandable and tailor the same to address the immediate and delayed health concerns.

\section{Objectives}

1. To critically review the gender sensitivity of tobacco control measures in India

2. To conduct a comparative analysis of gender responsive strategies in India with those in smokeless tobacco high burden countries

3. To make practical, feasible recommendations to enhance gender responsiveness of tobacco control measures in India in general and smokeless tobacco in particular

\section{Methodology}

We carried out a comprehensive literature review attempting to capture key information on gender responsiveness/sensitivity of strategies for tobacco control in general and for smokeless tobacco control in particular in India. The search terms were "tobacco control, smokeless tobacco, programme, policy, AND gender sensitivity, gender focus, gender responsive" in combination and as single terms. We drew key information related to gender focus in tobacco related policies/ programmes/strategies/actions from published articles/ and electronic data sources such as PubMed, Wiley, and ProQuest, programme documents and reports published by national governments and international agencies from websites and other online sources. Inclusion criteria were the presence of discussion on gender responsiveness/ sensitivity and tobacco control (smokeless tobacco particularly), reference to smokeless tobacco high burden countries, and publication in English within the last 20 years. Information was extracted of terms or conceptual description related to gender emphasis, gender analysis, gender responsive actions/messages to minimise exposure, impact of marketing on women and girls, counter measures for gender gaps in programme delivery, gender sensitive service delivery in India vis-à-vis the other smokeless tobacco high burden countries.

\section{Study Findings and Discussion}

Our search yielded 35 papers and reports from India describing policies relevant to SLT and women. Out of these, 6 are policy documents and 29 are papers and reports.

The documents report that smokeless tobacco products vary greatly in types, constituents, packaging, forms, addiction, and harm potential. It is obvious that these are challenging to study and control owing to the diversity. They are usually cheaper than the smoked forms. Smokeless tobacco products are often produced in small cottage industries and sold in unregulated markets. There is an urgent need to develop public health initiatives to minimize tobacco use among women in LMICs.

The advocacy for tobacco control by the civil society and community led initiatives has acted in synergy with tobacco control policies of the Government. It is evident that tobacco and smokeless tobacco related information and awareness activities need to focus more on women. This much needed change, would receive impetus with 
revisions in Governmental tobacco control policies.

The influences related to sex/gender specific issues on womens' tobacco use have received some importance since the 1980s. The consideration traditionally was to pregnancy and reproduction related issues to smoking; attention to other health issues and risk factors is a recent phenomenon. The vigorous targeting of young girls and women, especially the disadvantaged groups by tobacco industry is real (Greaves et al., 2006).

The impact of tobacco on womens' health is also much more than that in men. The relative risk of oral cancer among women SLT users is 8 times higher than that for men, and the relative risk of cardiovascular disease among women SLT users is 2-4 times higher than in men. Relative risk of all-cause mortality due to SLT use is higher among women than among men. Three large cohort studies from India have shown a higher age-adjusted relative risk of death among SLT users. Corroborating this, four large studies in Western countries (two from Sweden and two from the United States) have also shown significantly higher mortality in SLT users (Gupta et al., 2016). In a country like India, where SLT use is high among women, the SLT products which are usually produced in the unorganized sector escape regulations unlike the smoked products and are freely available to women (Yadav et al., 2020).

India has been a leader in global tobacco control. With the increasing evidence of harmful and hazardous effects of tobacco, the Government of India has enacted legislations and comprehensive tobacco control measures. The Cigarettes Act (Regulation of Production, Supply and Distribution) in 1975 (Cigarettes Act, 1975) was one of the earliest in the series.

The statutory warning "cigarette smoking is injurious to health" was mandatorily displayed on all cigarette packages, cartons and advertisements of cigarettes. Other Acts promulgated include Prevention of Food Adulteration Act (PFA) (Amendment) 1990 (PFA, 1990) the Drugs and Cosmetics Act 1940 (Amendment), which banned inclusion of tobacco in all dental products (Drugs and Cosmetics, 1940), Cigarettes and Other Tobacco Products (Prohibition of Advertisement and Regulation of Trade and Commerce, Production, Supply and Distribution) Act (COTPA), in 2003 (COPTA, 2003) . The law pertaining to pictorial warnings on tobacco products packages was implemented in 2009. To strengthen implementation of the tobacco control provisions under COTPA and policies of tobacco control mandated under the WHO FCTC, the Government of the India piloted National Tobacco Control Programme (NTCP) in 2007-2008 (NTCP, 2007). The NTCP focusses on various aspects of Tobacco control at national, state and district levels. Although initially focused on cigarette smoking, regulation of SLT products has also increased in recent years.

In spite of the tremendous political will and strong legal framework for tobacco control, implementation of NTCP by states in terms of women centric actions and gender based strategies are non-existent. Educational and community oriented interventions focussing on women and girls are paramount.

Women-specific tobacco prevention education and cessation programmes need to be developed which clearly address the harms, misconceptions (natural product), and local concerns. There has been little integration of gender considerations in research, policy and programmes. Studies indicate that the recent change in India from a symbolic warning label (a scorpion) to graphic warning labels (graphic images of cancer of the mouth, jaw or neck) did not bring any improvement in health-related outcomes, such as increased awareness about its harms or reduction in tobacco use (Gravely et al., 2016)

Our work makes a case for gender and diversity analyses in smokeless tobacco control. In HICs tobacco control policies have had a major impact in reducing prevalence of smoking, although this reduction has not been equitable across population subgroups. It is observed that elements of comprehensive tobacco control policies that reduce overall tobacco use can have more limited impact among girls and women, particularly those of low socioeconomic status (Greaves et al., 2006).

An important step by Ministry of Labor was alternate livelihood initiatives. A series of training programmes were undertaken in bidi rolling areas to train women bidi rollers in alternate vocations. However, there is hardly any reference to smokeless tobacco or the gender specific steps to curb tobacco use.

Recent studies suggest that gender-targeted and/or gender-tailored tobacco cessation communications have potential to reach this vulnerable group (Campbell et al., 2019; Toll et al., 2008). Visible cessation messages in clinical and population settings may have the potential to motivate women to quit during and even before pregnancy. Even brief counselling for pregnant women about quitting was found to increase the odds of cessation by 1.7 (Melvin et al., 2000). Studies indicate that graphic warning labels (GWLs) with images of women, babies, and tobacco-related disease were impactful for women from a vulnerable population with high smoking rates (Campbell et al., 2019). Under the NTCP, during efforts to disseminate knowledge and awareness of adverse impact of tobacco use, it was seen that nearly 45.3 per cent of women SLT users did not notice health-related warnings on SLT products, which was more than two times much higher than the male counterparts. (Singh et al., 2020)

The World Health Organization (WHO) introduced the MPOWER package (an acronym representing six evidence-based control measures)( World Health Organization, and Research for International Tobacco Control., 2008) to support policy implementation under the Framework Convention on Tobacco Control (FCTC).

In the study by Ngo et al., (2017) (the effect of MPOWER ) policies on smoking prevalence and cigarette consumption in a global context was examined. When the impacts were measured using percent changes, one-unit increase in the MPOWER composite score decreased smoking prevalence by 0.8 percent though this association was not seen for female smoking prevalence.

In an assessment of WHO MPOWER package among women, the magnitude of effect of different policy indicators varied across age groups. For those aged over 15, each 1-point increment in W (Warning about dangers) and $\mathrm{E}$ (Enforcement of ban) scores was independently 
associated with an average reduction in prevalence by $3.6 \%(95 \%$ UI, -4.5 to $-2.9, \mathrm{P}<0.0001)$ and $1.9 \%(95 \%$ $\mathrm{UI},-2.9$ to $-1.8, \mathrm{P}=0.002)$, respectively. These findings were similar across age groups. Smoking ban (P) scores were not associated with reduced prevalence among women aged 15-29 years or over 50 years. However, a 1-unit increase in $\mathrm{P}$ scores was associated with a $1.3 \%$ ( $95 \% \mathrm{UI},-2.3$ to $-0.2, \mathrm{P}=0.016$ ) reduction in prevalence among women aged 30-49 years (Flor et al., 2021).

There is evidence that certain elements of tobacco control policies that play a role in reducing overall smoking can have limited impact among girls and women, particularly those of low socioeconomic status (Greaves et al., 2007). Generally, women are less likely to smoke in public places, whereas men might be more frequently influenced by smoking bans in public places like bars, restaurants, clubs, and workplaces a (Amos et al., 2012). Including best practice health warnings to unbranded packages might be an effective way of informing about the negative effects of smoked as well as smokeless tobacco use while also mitigating the tobacco industry's marketing efforts of using cigarette packages to make these products more appealing, especially for women and young people (Wakefield et al., 2015)

In a study involving Scheduled Tribal women from Karnataka, Placek et al planned to test an anti-tobacco messaging strategy aimed to compare a standard anti-tobacco messaging strategy about general health risks that tend to occur late in life with one that emphasized reproductive harms specific to women that tend to occur earlier in life. It was predicted that reproductive-aged women in a disadvantaged population exposed to information about the near-term reproductive harms of tobacco use would be more likely to (1) reduce tobacco use, (2) increase knowledge of tobacco harms, (3) report more intentions to quit and (4) be more likely to share this information with others compared with women exposed to information about generic harms of tobacco use that are not specific to reproductive-aged women and tend to occur far in the future. The women were randomly shown either a general harms presentation (GHP) or reproductive harms presentation (RHP). At baseline, participants were aware of general harms but not of reproductive harms. Both interventions increased knowledge of harms. RHP was not more effective in reducing tobacco use than the GHP. In the RHP group fetal health was particularly salient in discussions while that in GHP group, oral health was highly salient (Placek et al., 2021). This study brought out another important dimension of the relationship between iron deficiency and tobacco use because reproductive-aged Indian women and girls suffer from high rates of anemia and smokeless tobacco use among them is on the rise. These findings highlight the need for public health efforts to tackle anemia in conjunction with tobacco use in women, rather than investigating them as isolated health concerns. Public health interventions that emphasize more immediate harms to fitness might be more effective at changing behaviours than those that emphasize harms occurring late in life (Saad et al., 2006)

Studies confirm that pregnant women rely heavily on social learning to learn about toxic foods and substances that can disrupt fetal development during pregnancy (Placek et al., 2017). Therefore, peer-to-peer transmission of tobacco harms is an effective strategy in reducing use because peers are less intimidating than physicians (Williams et al., 2011)

Tobacco is not always viewed as harmful by women in LMICs, and is even thought to provide benefits, such as energy booster. The women reported that chewing tobacco was for its perceived positive benefits and because prominent members of the community were tobacco users (Placek et al., 2019). Sex-disaggregated prevalence use ratios vary by region and show a picture generally opposite to that of tobacco smoking: high income countries have a higher prevalence ratio of men compared to women (8:1), while in low-income, lower middle- and upper middle-income countries, the male to female prevalence ratio is between $1.7: 1$ and 1.9:1 (Secretariat of the WHO Framework Convention on Tobacco Control. 2018). In India, SLT use is common although smoking is unacceptable among several communities. The factors which were reported to be the reasons promoting SLT use in women are easy availability and low cost of SLT products. A key factor influencing SLT use among disadvantaged women using SLT was the desire to suppress hunger while performing difficult and laborious tasks (Gupta et al., 2016). Even when it comes to quitting SLT use, the demand from and supply of services to women remain much lower than men (Singh et al., 2020).

Last but not the least,just as the women and girls are continuously targeted by tobacco industry as their next generation smokers, there is the danger of them being focussed for smokeless products. The tobacco companies have had a longstanding awareness that mildly flavoured tobacco products would be preferred by non-users and the young by evoking a perception of mildness and blinding the strong tobacco taste and unpleasant mouth feel. Flavoured tobacco products are slowly but surely increasing their market share and the industry is aggressively marketing them (Kostygina et al., 2016).

SLT use receives much less attention than their smoked counterparts in policies and programmes. Women use SLT more than smoked products. SLTs produce significant impact on womens' health by causing oral and other cancers, adverse reproductive outcomes, nutritional deficiencies etc. This information has not translated to any specific policy observations or subsequent actions (Singh et al., 2020; Yadav et al., 2020). India's tobacco control efforts must be lauded for the fall in prevalence of tobacco use, including SLTs (GAT-2 2017), however, they have focussed on smoked products more and have been gender neutral.

Recommendations Greater focus on women and also SLT use use is urgently required for curb tobacco use Myths regarding SLT products should be dispelled by implementing large and clear pictorial warnings, educational, and mass media drives. Stringent ban on tobacco-related advertising, promotion, and sponsorship for SLT with strict implementation of legislation related 
to the sale of tobacco to/by minors should be considered. Currently, anti-tobacco efforts in India largely target commercial products, which are not typically used by Indian women. By not including "natural" products in anti-tobacco use interventions, women might perceive these substances as being safe to consume (Banerjee et al., 2014).

In conclusion, SLT has received less attention over the years compared to its smoked counterparts. The determinants and implications of SLT use in women are multifold and need more nuanced approach and analysis for holistic, effective control measures. The women centric issues related to SLT use which need to be explored for policy and programmatic addressal include increased use across all ages compared to smoking, easy availability and low pricing, preference of type of SLT, socio economic influencers, livelihoods, disproportionate impact on health and mortality, access to information and services as well as the threat of an aggressive tobacco industry. . It is high time that tobacco control policies in India adopted a gender focussed approach acknowledging the implications of SLT on the population in general and women in particular.

\section{Author Contribution Statement}

The authors confirm contribution to the paper as follows: study conception and design: AGR,PGS,SBN; data collection: AGR, PGS; analysis and interpretation of results: AGR, PGS, SBN. Author; draft manuscript preparation: AGR,PGS. All authors reviewed the results and approved the final version of the manuscript.

\section{Acknowledgements}

The publishing cost was provided by WHO office in South-East Asia.

\section{References}

Amos A, Greaves L, Nichter M, Bloch M (2012). Women and tobacco: a call for including gender in tobacco control research, policy and practice. Tob Control, 21, 236-43.

Arora M, Tewari A, Tripathy V, et al (2010). Community-based model for preventing tobacco use among disadvantaged adolescents in urban slums of India. Health Promot Int, 25, 143-52.

Banerjee SC, Ostroff JS, Bari S, et al (2014). Gutka and Tambaku paan use among South Asian immigrants: a focus group study. J Immigr Minor Health, 16, 531-9.

Campbell BK, Le T, Yip D, et al (2019). Sex differences in graphic warning label ratings by addictions clients. $T o b$ Regul Sci, 5, 3-14.

Flor LS, Reitsma MB, Gupta V, Ng M, Gakidou E (2021). The effects of tobacco control policies on global smoking prevalence. Nat Med, 27, 239-43.

Government of India. Drugs and Cosmetics Act 1940 (Amendment) 1992.

Government of India. GATS-2 Global Adult Tobacco Survey. Fact Sheet. Delhi, India: Ministry of Health and Family Welfare Government of India. 2017.

Government of India. National Tobacco Control Programme, 2007-08.

Government of India. Prevention of Food Adulteration Act (PFA) (Amendment) 1990
Government of India. The Cigarettes Act (Regulation of Production, Supply and Distribution) 1975.

Gravely S, Fong GT, Driezen P, et al (2016). An examination of the effectiveness of health warning labels on smokeless tobacco products in four states in India: findings from the TCP India cohort survey. BMC Public Health, 16, 1-11.

Greaves L (2007). Gender, equity and tobacco control. Health Sociol Rev, 16, 115-29.

Greaves L, Jategaonkar N (2006). Tobacco policies and vulnerable girls and women: toward a framework for gender sensitive policy development. J Epidemiol Commun Health, 60, 57-65.

Greaves L, Johnson J, Bottorff J, et al (2006). What are the effects of tobacco policies on vulnerable populations?. Can J Public Health, 97, 310-15.

Gupta PC, Arora M, Sinha D, Asma S, Parascondola M (2016). Smokeless tobacco and public health in India. Ministry of Health \& Family Welfare, Government of India: New Delhi. https://www.who.int/fctc/cop/sessions/cop8/ GenderResponsive-Tobacco-Control.pdf.

IARC Working Group on the Evaluation of Carcinogenic Risks to Humans, World Health Organization, \& International Agency for Research on Cancer(2007). Smokeless tobacco and some tobacco-specific N-nitrosamines (Vol. 89). World Health Organization.

Kostygina G, Ling PM (2016). Tobacco industry use of flavourings to promote smokeless tobacco products. $T o b$ Control, 25, 40-9.

Kyaing NN, Islam MA, Sinha DN, Rinchen S (2011). Social, economic and legal dimensions of tobacco and its control in South-East Asia region. Indian J Public Health, 55, 161.

Mehrotra R, Yadav A, Sinha DN, et al (2019). Smokeless tobacco control in 180 countries across the globe: call to action for full implementation of WHO FCTC measures. Lancet Oncol, 20, e208-e17.

Melvin CL, Dolan-Mullen P, Windsor RA, Whiteside HP, Goldenberg RL (2000). Recommended cessation counselling for pregnant women who smoke: a review of the evidence. Tob Control, 9, 80-4.

Ngo A, Cheng KW, Chaloupka FJ, Shang C (2017). The effect of MPOWER scores on cigarette smoking prevalence and consumption. Prev Med, 105, 10-4.

Onoh I, Owopetu O, Olorukooba AA, Umeokonkwo CD, Dahiru T, Balogun MS (2021). Prevalence, patterns and correlates of smokeless tobacco use in Nigerian adults: An analysis of the Global Adult Tobacco Survey. PLoS One, 16, e0245114.

Placek CD, Madhivanan P, Hagen EH (2017). Innate food aversions and culturally transmitted food taboos in pregnant women in rural southwest India: separate systems to protect the fetus?. Evol Hum Behav, 38, 714-28.

Placek CD, Magnan RE, Srinivas V, et al (2021). The impact of information about tobacco-related reproductive vs. general health risks on South Indian women's tobacco use decisions. Evol Hum Sci, 3, e4

Placek C, Roulette C, Hudanick N, et al (2019). Exploring biocultural models of chewing tobacco and paan among reproductive-aged women: Self-medication, protection, or gender inequality?. Am J Hum Biol, 31, e23281.

Raute LJ, Pednekar MS, Gupta PC (2009). Pictorial health warnings on cigarette packs: A population based study findings from India. Tob Use Insights, 2, TUI-S2751.

Saad G, Peng A (2006). Applying Darwinian principles in designing effective intervention strategies: The case of sun tanning. Psychol Marketing, 23, 617-38.

Siddiqi K, Shah S, Abbas SM, et al (2015). Global burden of disease due to smokeless tobacco consumption in adults: analysis of data from 113 countries. BMC Med, 13, 1-22. 
Singh S, Jain P, Singh PK, Reddy KS, Bhargava B (2020). White paper on smokeless tobacco \& women's health in India. Ind $J$ Med Res, 151, 513-21.

Sinha DN, Gupta PC, Kumar A, et al (2018). The poorest of poor suffer the greatest burden from smokeless tobacco use: A Study From 140 Countries. Nicotine Tob Res, 20,1529-32.

Sinha DN, Gupta PC, Ray C, Singh PK( 2012). Prevalence of smokeless tobacco use among adults in WHO South-East Asia. Indian J Cancer, 49, 342-6.

Sreeramareddy CT, Pradhan PMS, Mir IA,Sin S (2014). Smoking and smokeless tobacco use in nine South and Southeast Asian countries: prevalence estimates and social determinants from Demographic and Health Surveys. Popul Health Metrics, 12, 22.

The Cigarettes and Other Tobacco Products (Prohibition of Advertisement and regulation of Trade and Commerce, production, Supply and Distribution) Act, 2003.

Toll BA, Salovey P, O’Malley SS, et al (2008). Message framing for smoking cessation: the interaction of risk perceptions and gender. Nicotine Tob Res, 10, 195-200.

WakefieldM, Coomber K, Zacher M, et al (2015). Australian adult smokers' responses to plain packaging with larger graphic health warnings 1 year after implementation: results from a national cross-sectional tracking survey. Tob Control, 24, 17-25.

WHO FCTC Secretariat. Hawkes S, Buse K, Soon SY. Tob Control: Evidence and options for policies and programmes. Available from https://www.who.int/fctc/cop/sessions/cop8/ Gender-Responsive-TobaccoControl.pdf.

Williams JM, Dwyer M, Verna M, et al (2011). Evaluation of the CHOICES program of peer-to-peer tobacco education and advocacy. Community Ment Health J, 47, 243-51.

World Health Organization, \& Research for International Tobacco Control. (2008). WHO report on the global tobacco epidemic, 2008: the MPOWER package. Available: http:// monographs. iarc. fr/ ENG/ Monographs/ smokeless tobacco and some tobacco- specific N -Nitrosamines, 200789.

Yadav A, Singh PK, Yadav N, et al (2020). Smokeless tobacco control in India: policy review and lessons for high-burden countries. BMJ Global Health, 5, e002367.

\section{(ब) $(0 \otimes$}

This work is licensed under a Creative Commons AttributionNon Commercial 4.0 International License. 